\title{
Reliability-Based Analytic Models for Fatigue Lifetime Distribution Estimation of Series Mechanical Systems under Random Load considering Strength Degradation Path Dependence
}

\author{
Peng Gao ${ }^{1,2}$ and Liyang Xie ${ }^{3}$ \\ ${ }^{1}$ School of Mechanical Engineering, Liaoning Shihua University, Liaoning 113001, China \\ ${ }^{2}$ School of Mechanical Engineering and Automation, Zhejiang Sci-Tech University, Hangzhou 310018, China \\ ${ }^{3}$ School of Mechanical Engineering and Automation, Northeastern University, Liaoning 110819, China \\ Correspondence should be addressed to Peng Gao; gaopeng@lnpu.edu.cn
}

Received 13 August 2017; Accepted 16 October 2017; Published 14 November 2017

Academic Editor: Roman Wendner

Copyright ( $\odot 2017$ Peng Gao and Liyang Xie. This is an open access article distributed under the Creative Commons Attribution License, which permits unrestricted use, distribution, and reproduction in any medium, provided the original work is properly cited.

Time-dependent statistical characteristics of load process and strength degradation process are critical to lifetime distribution of series mechanical systems. Conventional rain-flow counting method for lifetime distribution estimation has limited practical application due to its strict requirement for statistical properties of load process. Besides, dynamic interaction between load process and strength degradation process results in strength degradation path dependence (SDPD). SDPD and failure dependence of components jointly bring considerable difficulties in prediction of system lifetime distribution and system residual lifetime distribution. To address these problems, reliability-based analytic models for estimation of whole lifetime distribution and residual lifetime distribution of series mechanical systems under random load are developed in this paper, which take the time-dependent statistical parameters of load process and strength degradation process as the input of the models. Furthermore, SDPD and failure dependence of components are taken into account in the proposed models in an explicit mathematical expression. The results show that SDPD, failure dependence of components, and initial strength dispersion have significant influences on system lifetime distribution.

\section{Introduction}

With the function, structure, and working environment of mechanical products becoming complicated, accurate assessment of fatigue lifetime distribution of mechanical systems in their full lifetime cycles is encountered with new challenges and has attracted extensive attention from researchers and engineers [1-3]. Various random factors, such as randomness in material, geometry, and manufacturing of components and working load, make fatigue lifetime distribution analysis more complex and difficult. In the field of mechanical engineering, analytic methods for quantitative estimation of a systematic fatigue lifetime distribution are mainly based on lifetime distributions of components in the system and the systematic structure function. Lifetime distributions of mechanical components are obtained from fatigue tests, which are usually performed under load with constant amplitude. To consider the randomness of working load, rainflow counting (RFC) method is widely used in fatigue tests. Current fatigue test methods provide an important foundation for lifetime evaluation of mechanical products [4].

Some innovative research has been carried out for the random lifetime distribution estimation with some specified distribution types. For instance, Elperin and Gertsbakh developed a method for estimating the exponential mean lifetime in a random-censoring model with incomplete information. In the proposed method, the maximum-likelihood method and the Monte Carlo simulations are combined to obtain the point and interval estimates of the mean value of the lifetime [5]. Pan et al. suggested using a multivariate 


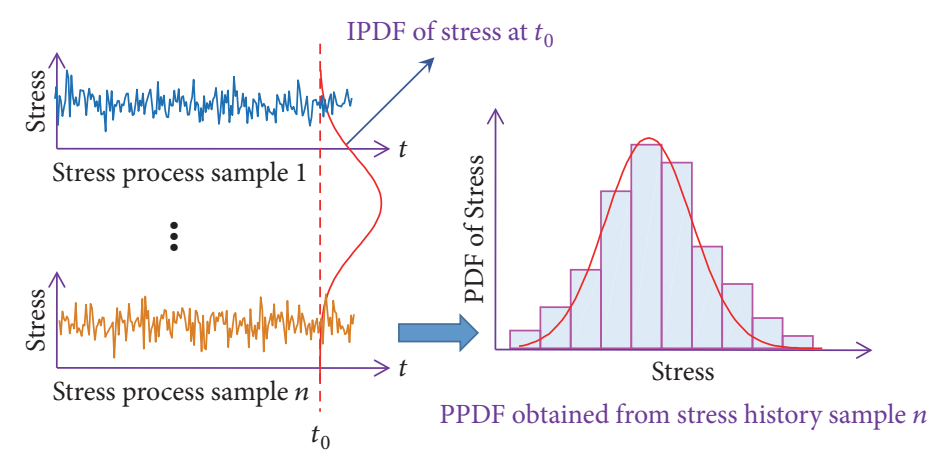

Figure 1: Different expressions of load PDFs.

Birnbaum-Saunders distribution and its marginal distributions for lifetime distribution analysis of systems that have multiple dependent performance characteristics. In the proposed models, the system degradation was assumed to follow the gamma processes [6]. Asgharzadeh et al. presented a method for two-parameter bathtub-shaped lifetime distribution estimation based on upper record values by constructing exact confidence intervals and exact joint confidence regions of parameters [7]. Furthermore, confidence intervals were estimated via the asymptotic normality of the maximumlikelihood estimators. Kayid and Izadkhah analyzed the random lifetime distribution by using the order statistics theory and assuming that the residual lifetime followed the exponential distribution [8]. Gong et al. proposed a method for fatigue life estimation of the screw blade in the screw sand washing machine. The random load was assumed to follow the Gauss distribution [9]. Moreover, in the statistics of random load, the Markov chain method, Monte Carlo simulations, and the finite element method were combined for the purpose of reducing research cost. Then, the rain-flow counting method, the Goodman stress correction method, and Miner's rules were jointly used to assess lifetime distribution of the screw blade.

In some circumstances, difficulties could be encountered in the process of systematic lifetime distribution modeling, which are listed as follows, and there needs an extension of current modeling methods to overcome these difficulties.

(1) For mechanical components, fatigue tests under load with constant amplitude are aimed at obtaining lifetime distributions resulting from the randomness of material parameters. The RFC method is used to deal with the randomness of load. Material parameters, such as strength, could exhibit a dynamic stochastic degradation process which is governed by the time-dependent statistical properties of load $[10,11]$. According to the stochastic process theory, the randomness of load can be expressed by two methods: (a) the probability density function (PDF) at each moment or at each load application, which is always mathematically expressed via a stochastic process and is referred to as instantaneous pdf (IPDF) in this paper; (b) the PDF obtained directly from a load history sample, which is referred to as the process PDF (PPDF) in this paper. The schematic illustration of IPDF and PPDF of load is shown in Figure 1. It can be learnt that the load PDF obtained by using the RFC method is essentially a PPDF.
Unless the assumption that the load process is ergodic and stationary is strictly satisfied, IPDFs cannot be approximated to PPDF. When the random load, characterized by PPDF from RFC method, is applied on the specimen in the fatigue tests, it infers that the IPDF is approximated by PPDF and the information about the time dependence characteristics of IPDF of the stochastic load process might be lost, which could result in the error in the assessment of lifetime distribution and will be explained in detail in Section 2. Therefore, it is of considerable importance to establish analytical models for lifetime distribution assessment of mechanical systems with the load process and strength degradation process taken as the input of the models and considering the dynamic interaction between the two processes.

(2) As mentioned above, fatigue tests are mainly aimed at lifetime distributions of components. Systematic lifetime distributions are calculated according to the system failure criterion or structure function. However, lifetime distributions of components in a mechanical system are always mutually statistically correlative in the process of transferring load or motion. Therefore, it is difficult to provide an explicit expression of the system structure function. Furthermore, the implicit structure function is time-dependent due to the time-dependent load process. The assumption that component lifetime distributions are mutually statistically independent could lead to an error in the systematic lifetime distribution estimation.

(3) For mechanical systems, residual lifetime analysis is quite vital to their fault diagnosis and maintenance. When considering the statistical dependence of component lifetime distributions and the complexity of the stochastic load process, it is necessary to improve the conventional methods for residual lifetime analysis which are always performed based on independent component lifetime distributions.

To address the aforementioned problems, analytic models for fatigue lifetime distribution estimation of mechanical systems under random load are developed in this paper, which can take the time-dependent statistical characteristics of load and the failure dependence of components into consideration and provide an explicit expression of the PDF of systematic lifetime that characterized the systematic lifetime distributions. Moreover, residual lifetime models of dependent mechanical systems are established. Besides, numerical examples are given to validate the proposed models and 
illustrate key factors that have great influences on system lifetime distributions.

\section{Randomness and Dependence of Fatigue Lifetime Distributions of Components}

For electronic elements, lifetime always follows the exponential distribution, which is represented by the parameter of constant failure rate [12]. The assumption of constant failure rate about the electronic elements facilitates the regular lifetime tests or the accelerated lifetime tests. However, for mechanical components, time-dependent random vibration load, random impact load, and randomness in the material parameters caused by manufacturing are always encountered in practice [13-15]. Moreover, the time-dependent stochastic load process and the corresponding time-dependent degradation process of the material parameters are mutually statistical correlative. Meanwhile, the lifetime distributions of mechanical components are quite sensitive to these random factors in the working load and the material parameters. Therefore, sometimes, it is impractical to analyze the impacts of these random factors on the lifetime distributions of mechanical components and the systems only via fatigue tests. It is important to develop analytical models of lifetime distributions of mechanical components and mechanical systems, which takes these random factors as their input parameters and could be used to provider further information for improvement of design and manufacturing.

\subsection{Influence Factors of Component Lifetime Distributions. As} a matter of fact, a PPDF is a comprehensive embodiment of all the IPDFs within the time period when PPDF is measured. Only when the assumption that the load is ergodic and stationary is strictly satisfied, PPDF steadily approximate to IPDF. Otherwise, the PPDF mathematically have no direct functional relationship with IPDFs and the IPDF cannot be approximated by PPDF. RCF method is used to acquire a PPDF of load. When the load is not ergodic or stationary, fatigue tests based on a PPDF by using the RFC method could lead to error in fatigue lifetime distributions. Furthermore, the statistical characteristics of strength degradation process are determined by the stochastic load process. The two correlative stochastic processes both contribute to the lifetime distribution of components. The error in component lifetime distribution mentioned above due to the incorrect usage of RFC method could be magnified by the complex interaction between the two correlative stochastic processes.

For instance, suppose that the residual strength (MPa) of the components under load application for $n$ times is expressed as follows:

$$
r(n)=r\left(1-\sum_{i=1}^{n} \frac{x_{i}^{2}}{10^{9}}\right),
$$

where $x_{i}$ is the magnitude of load at the $i$ th load application and $r$ is the initial strength that is equal to $450 \mathrm{MPa}$. In the load process, the magnitude of load at each load application follows the normal distribution with the standard deviation of $30 \mathrm{MPa}$ and the mean value of $\mu_{s}(n)$ (MPa) expressed as follows:

$$
\mu_{s}(n)=200(1+0.001 n) .
$$

The actual working process is simulated by the Monte Carlo simulations (MCS) with the flow chart shown in Figure 2. In Figure 2, the load process is generated according to the given load IPDF and the PDF of the component lifetime is shown in Figure 3. Besides, the PPDF of load is obtained via the statistics of the simulated IPDF of load in Figure 2. Then, with the PPDF taken as the approximate IPDF, the PDF of the component lifetime according to the flow chart in Figure 2 is shown in Figure 3.

From Figure 3, it can be seen that it could result in large error in lifetime distribution estimation to approximate the IPDF by PPDF due to neglecting the information of timedependent statistical characteristics of load. Thus, attention should be paid to the stochastic characteristics of load process when using the RCF method for lifetime assessment of mechanical components. Furthermore, it is necessary to develop models for lifetime distribution estimation with the time-dependent statistical characteristics of load considered, taken as the input of the models.

In addition, to consider the influences of the randomness of strength degradation process on the component lifetime distribution, suppose the initial strength $r$ follows the normal distribution with the mean value of $450(\mathrm{MPa})$. In the case where the standard deviation of $r$ is $10 \mathrm{MPa}, 20 \mathrm{MPa}$, and $30 \mathrm{MPa}$, respectively, the PDF of the component lifetime based on IPDF of load and that based on PPDF of load are shown in Figures 4 and 5, respectively.

From Figures 4 and 5, it can be learnt that, besides the statistical characteristics of load process, the statistical characteristics of material parameters have significant influences on component lifetime distribution. Moreover, the dynamic interaction between the load process and the stochastic process of material parameters makes it more difficult to acquire an accurate assessment of lifetime PDF. Therefore, it is necessary to develop a method for system lifetime distribution evaluation, which takes the stochastic load process, the stochastic degradation process of material parameters, and their interaction into consideration.

\subsection{Dependence of Component Lifetime Distributions.} Besides the factors that significantly influence component lifetime distributions mentioned in Section 2.1, the dependence of component lifetime distributions has great impacts on system lifetime distribution. Mechanical systems are designed for transferring load and motion. In the transferring process of load and motion, components in the system always share the common load sources. The strength degradation processes of different components in the system are mutually statistically dependent because of their interaction with the common load sources. Thus, the system lifetime distribution cannot be calculated based on the systematic failure criterion and the lifetime distributions of components obtained from independent fatigue tests. To illustrate the influences of the dependence of component 


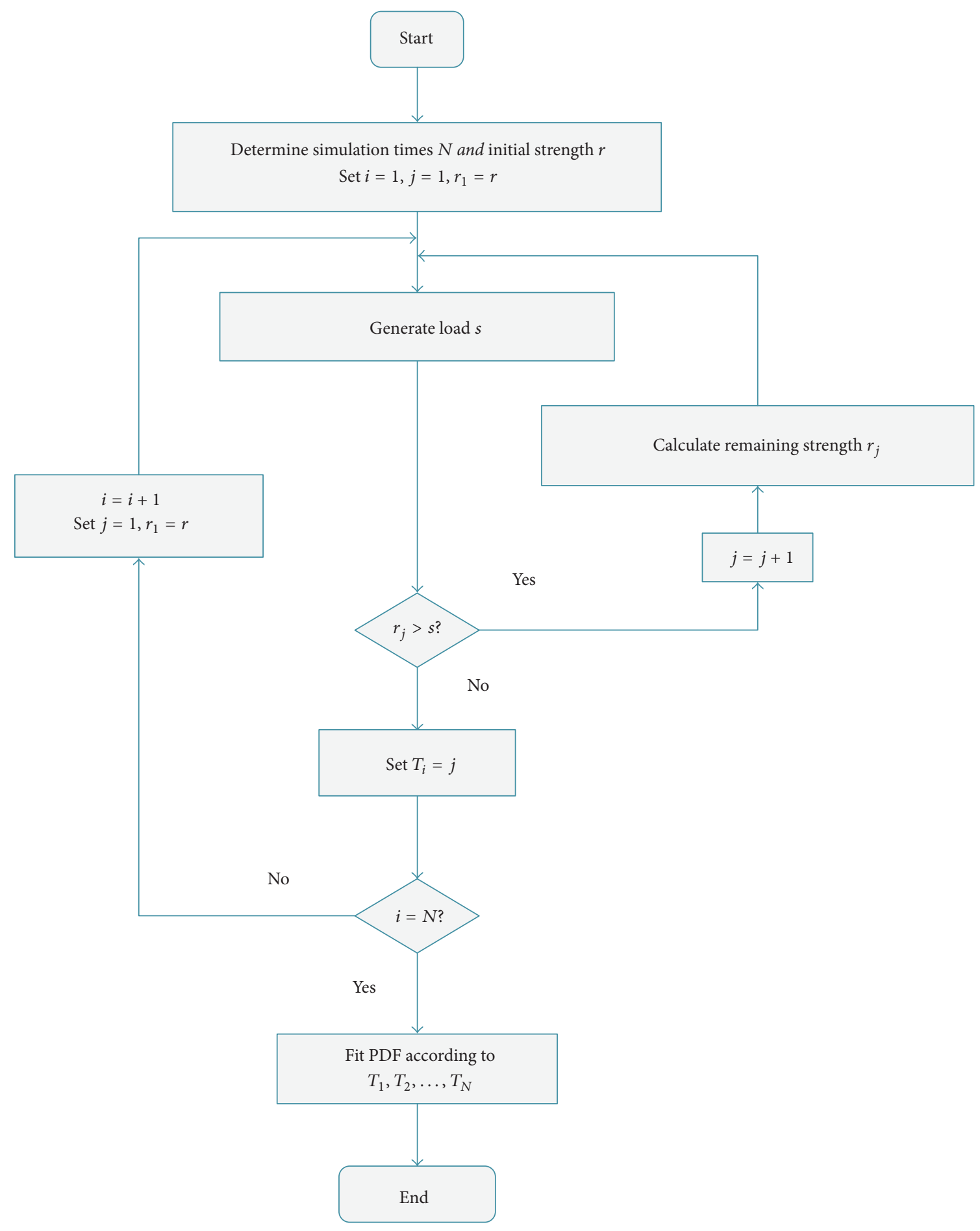

FIGURE 2: Flow chart for component PDF fitting.

lifetime distributions on system lifetime distribution, a numerical example is given as follows. The series mechanical system is composed of two components with the statistical properties of the load and the material parameters identical with those listed in Section 2.1. The standard deviation of the initial strength is $10 \mathrm{MPa}$. The flow chart of the simulation for lifetime PDF of an independent series system, in which the two components are mutually independent, is shown in Figure 6. Besides, when the two components share the same load, which constitute a dependent series system, the flow chart of simulation for lifetime PDF of the dependent system is shown in Figure 7. The results from the two simulations based on IPDF of load and the simulation results based on PPDF of load are shown in Figures 8 and 9, respectively. 


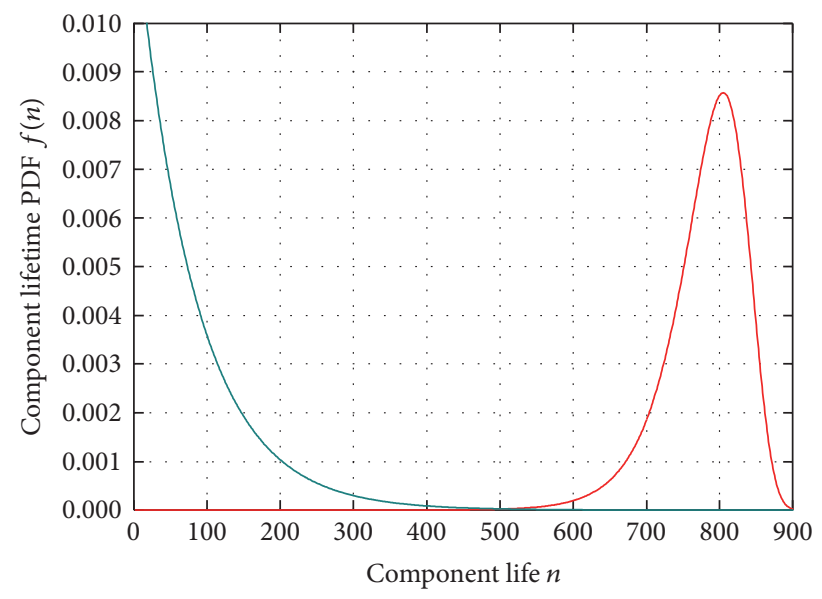

- IPDF-based life distribution PPDF-based life distribution

FIGURE 3: Lifetime PDF of components.

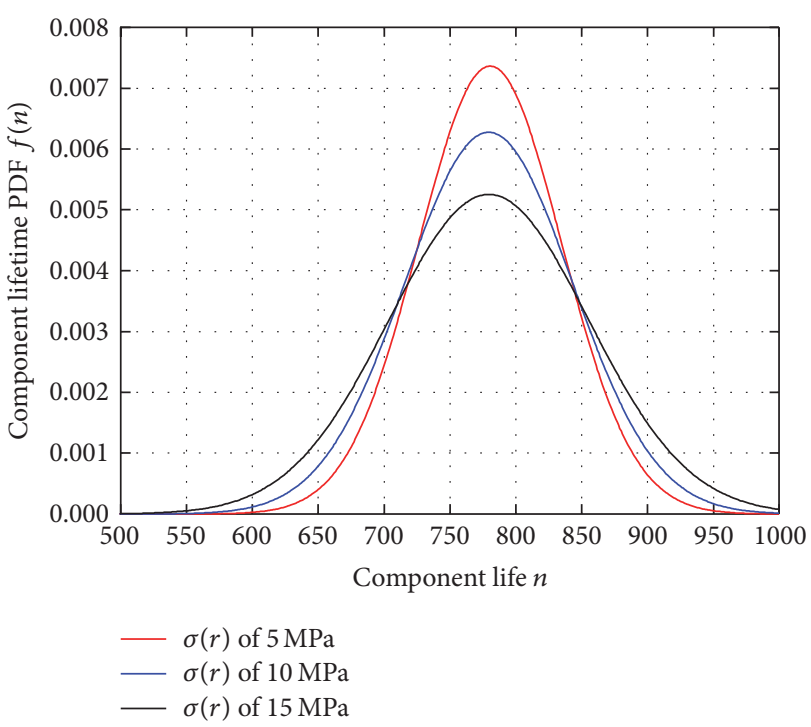

FIgURE 4: Lifetime PDF of components based on IPDF of load.

The failure dependence of components brings large difficulty in system lifetime distribution estimation due to the implicit system structure function. From Figures 8 and 9, it can be seen that the time-dependent statistical characteristics of load process have great influences on system lifetime distribution. Conventional RCF method for component and system lifetime distribution analysis should be used with caution. Compared with the component lifetime PDF, the system lifetime PDF shifts towards the left direction and the dispersion of the lifetime PDF decreases. Besides, the lifetime of the dependent system is distributed in the interval of longer lifetime compared with the lifetime of the independent system.

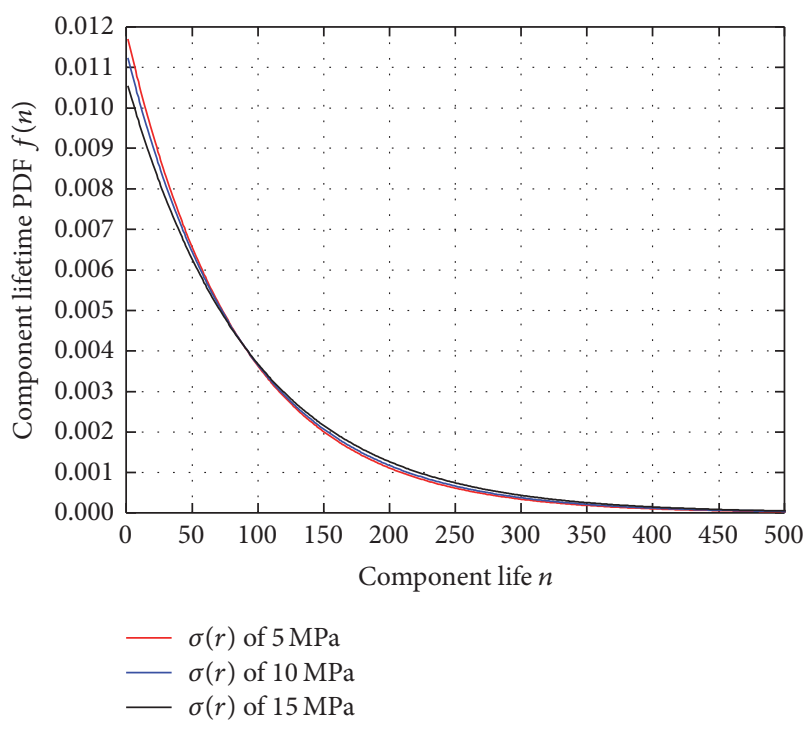

FIgURE 5: Lifetime PDF of components based on PPDF of load.

\section{Reliability-Based Analytic Models for Systematic Lifetime Distribution}

3.1. Whole Lifetime Distribution Models of Mechanical Systems. To take into account the interaction between the load process and strength degradation process and consider the dependence of component lifetime distribution, reliabilitybased analytic models for systematic lifetime distribution are developed in this paper. The proposed models are established according to the relationship between dynamic reliability and the failure probability. According to the reliability theory, the PDF of the system lifetime can be expressed as follows:

$$
f(t)=\frac{\partial}{\partial t}(1-R(t))=-\frac{\partial}{\partial t} R(t),
$$

where $R(t)$ is the dynamic reliability of mechanical systems. Thus, the lifetime distribution of mechanical systems, characterized by the PDF, can be obtained from the dynamic reliability function. Theoretically, the system reliability $R(t)$ can be calculated based on the component reliability and the system failure criterion. For instance, in a mechanical system with $k$ components, the reliability of the components are denoted by $R_{i}(t)(i=1,2,3, \ldots, k)$. Then, the PDF of a series mechanical system can be expressed as follows:

$$
f_{\text {series }}(t)=-\frac{\partial}{\partial t} R_{\text {series }}(t)=-\sum_{i=1}^{k} \frac{\partial}{\partial t} R_{i}(t) \prod_{j=1, j \neq i}^{k} R_{j}(t) .
$$

The PDF of a parallel mechanical system can be given by

$$
\begin{aligned}
f_{\text {parallel }}(t) & =-\frac{\partial}{\partial t} R_{\text {parallel }}(t) \\
& =-\sum_{i=1}^{k} \frac{\partial}{\partial t} R_{i}(t) \prod_{j=1, j \neq i}^{k}\left[1-R_{j}(t)\right] .
\end{aligned}
$$




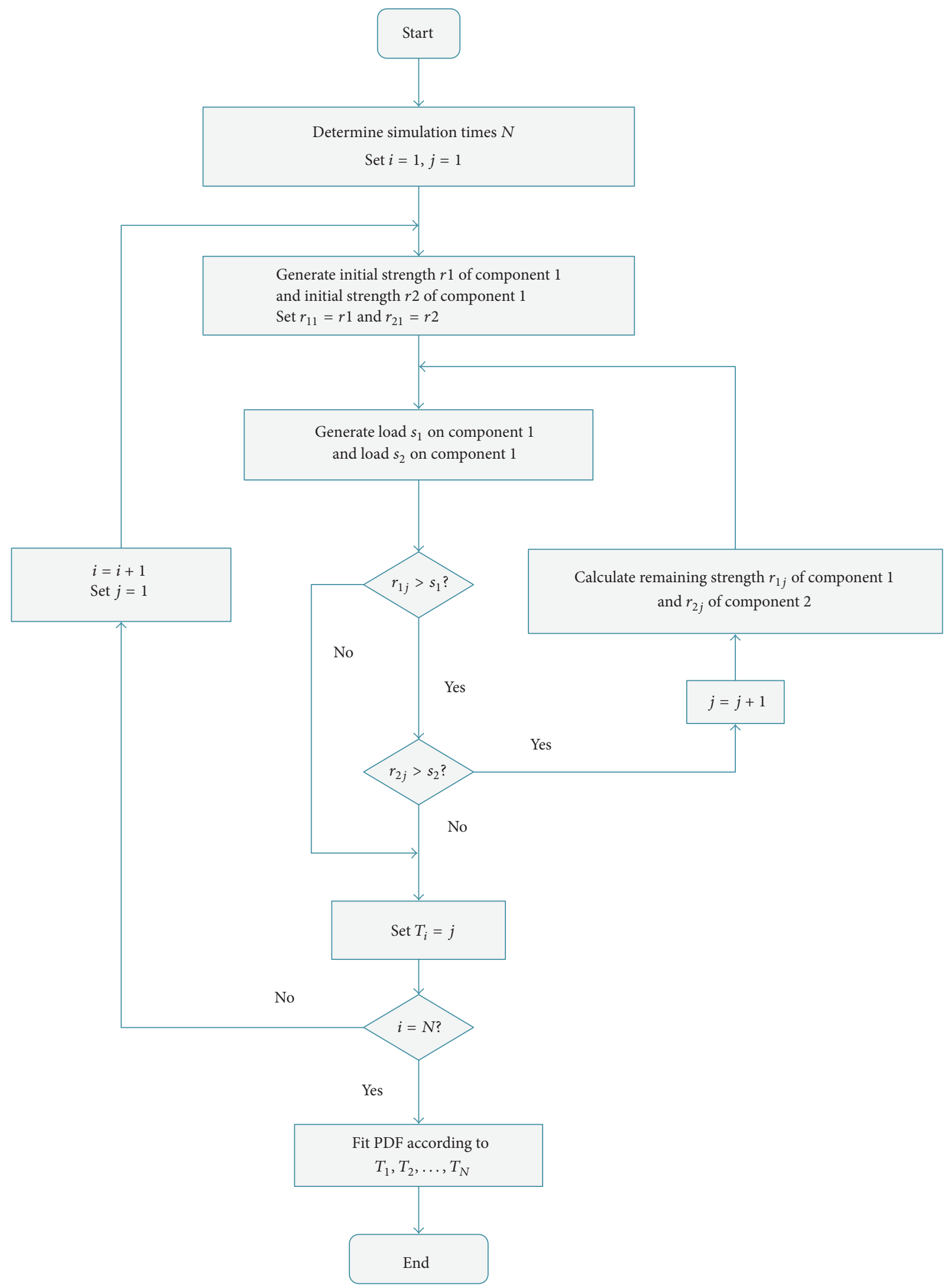

FIGURE 6: Flow chart for PDF fitting of the independent system. 


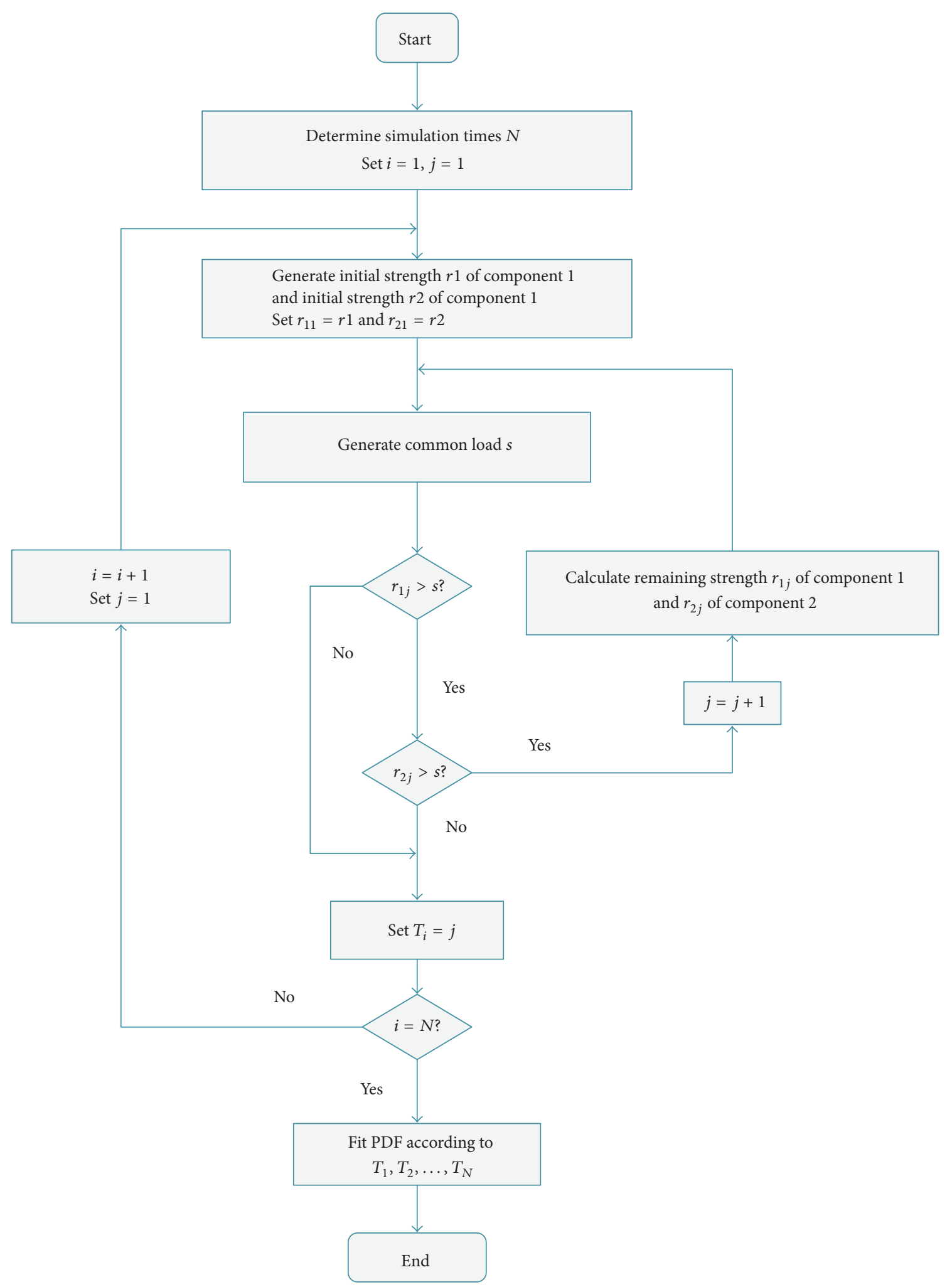

FIGURE 7: Flow chart for PDF fitting of the dependent system. 


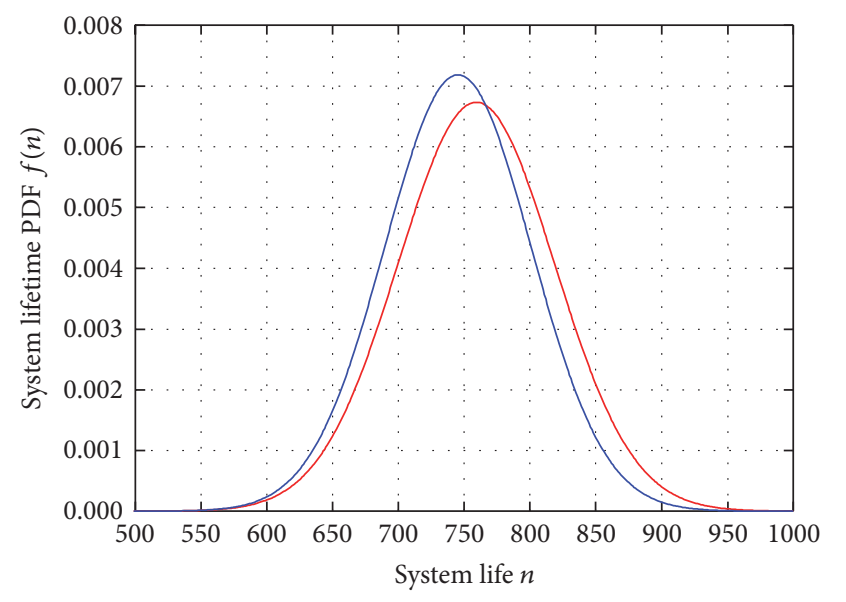

- Dependent system

— Independent system

Figure 8: Lifetime PDF of systems based on IPDF of load.

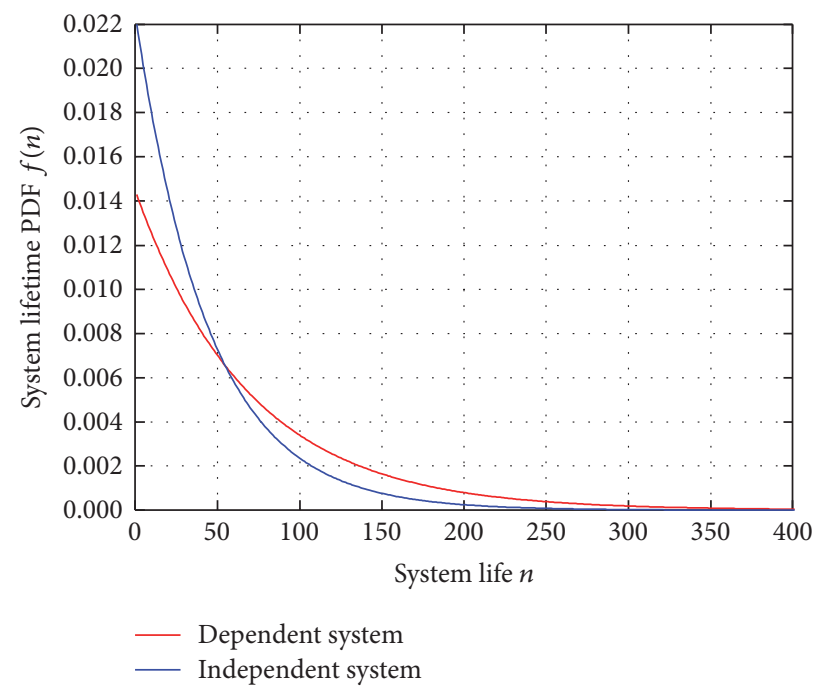

FIGURE 9: Lifetime PDF of systems based on PPDF of load.

Equations (4) and (5) are derived from conventional reliability theory. However, there exist some problems in the calculation of component reliability $R_{i}(t)$ and system reliability $R(t)$, which are listed as follows.

(1) As described above, in the case of complex stochastic load process, it is difficult to propose an appropriate fatigue test scheme to acquire the lifetime distribution of components. Consequently, the component reliability $R_{i}(t)$ cannot be calculated directly via the component lifetime distribution.

(2) Dynamic reliability models of mechanical components are mainly developed via extending the conventional moment-based reliability methods, such as the first-order second-moments (FOSM) method [16-20] and the secondorder second-moments (SOSM) method. In the conventional moment-based reliability models, reliability is calculated according to the performance function, which can be expressed as the difference between the strength $r$ and the load $s$ as follows:

$$
z=r-s
$$

It should be noted that, in (6), $s$ represents generalized load including force, stress, and temperature which is determined by the failure criterion of the components. When the static strength and load are independent variables that follow the normal distribution, reliability can be accurately computed via the mean value and the standard deviation of $z$ as follows:

$$
R=\Phi\left(\frac{\mu_{z}}{\sigma_{z}}\right),
$$

where $\Phi$ is standard normal distribution function. Provided that the condition of independent normal variables is not satisfied, a series of approximate reliability algorithm are proposed such as the aforementioned FOSM method and SOSM method. To calculate the dynamic reliability of components, static variables of $r$ and $s$ in (6) are replaced with the stochastic strength degradation process $r(t)$ and the stochastic load process $s(t)$ and (6) can be rewritten as

$$
z(t)=r(t)-s(t)
$$

Then dynamic reliability is further calculated according to $z(t)$. However, strength degradation path dependence (SDPD) is neglected when using this method, which could cause large calculation error as is described in the authors' former research [21].

To illustrate the phenomenon of SDPD in reliability calculation, two simulations are carried out. In the first simulation, which are independent of any analytical reliability models, practical operational processes of mechanical components are simulated with the flow chart shown in Figure 2. Besides, the distributions of residual strength at each load application are obtained by recording the residual strength in the first simulation, which is set as the input in the second simulation with the flow chart shown in Figure 10. Thus, the reliability obtained from the second simulation is based on the residual strength distribution at each load application, which is essentially the method expressed by (8). In the simulations, the deterministic strength degradation law is expressed by (1) and the initial strength follows the normal distribution with the mean value of $450 \mathrm{MPa}$ and the standard deviation of $20 \mathrm{MPa}$. The load at each load application follows the normal distribution with the mean value of $350 \mathrm{MPa}$ and the standard deviation of $20 \mathrm{MPa}$. The component reliability with the two simulations is shown in Figure 11.

It can be seen that it could result in computational error in reliability when directly using the strength distribution at each load application in (8) due to neglecting SDPD. The detailed explanation of the phenomenon of SDPD can be referred to in [21].

(3) Analogous to lifetime distribution of mechanical components, the component reliability is mutually statistically correlative. It is difficult to provide an explicit expression of the time-dependent system structure function for system reliability computation. 


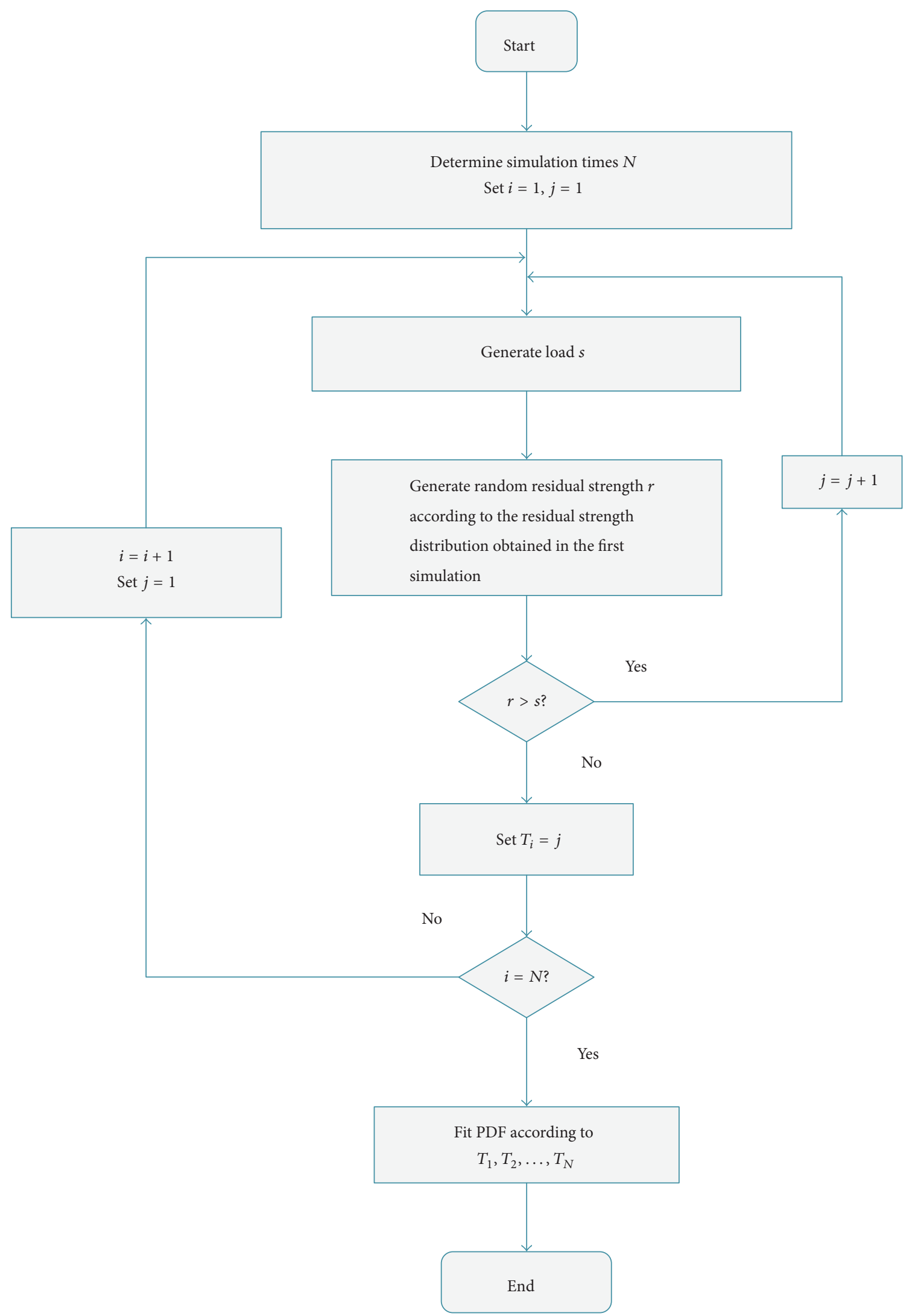

FIGURE 10: Flow chart for component reliability based on residual strength distributions. 


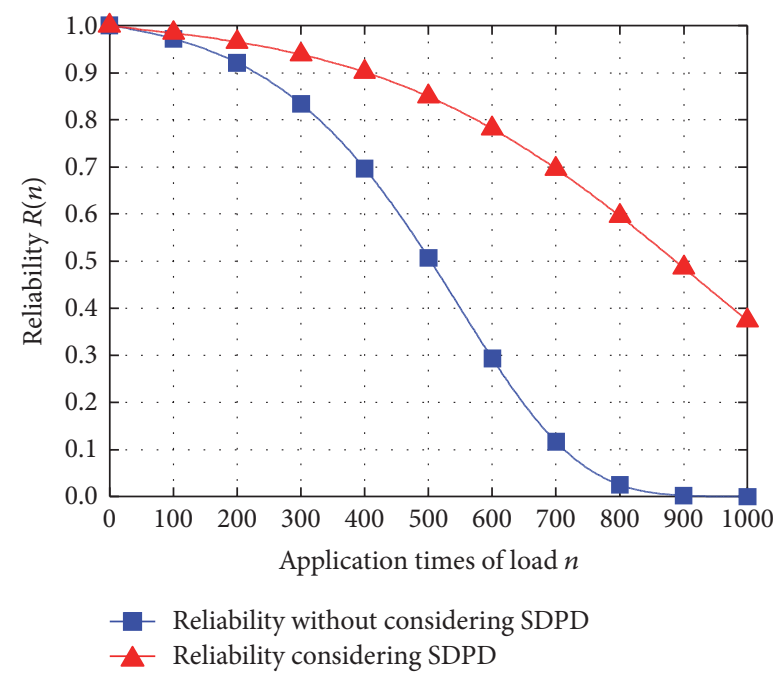

FIGURE 11: Comparison between reliability considering SDPD and reliability based on residual strength distributions.

To address the problems above and take the load process and the strength degradation process as the input of proposed models, the lifetime distribution models of series mechanical systems are established based on the dynamic reliability models developed in the authors' previous research [21]. For a series mechanical system with $k$ components, the dynamic reliability can be calculated by

$$
\begin{array}{r}
R(n)=\int_{0}^{\infty} k\left[1-\int_{0}^{r_{0}} f_{r_{0}}\left(r_{0}\right) d r_{0}\right]^{k-1} f_{r_{0}}\left(r_{0}\right) \\
\cdot\left\{\prod_{i=0}^{n-1}\left[\int_{0}^{r_{0}\left(1-i \int_{0}^{\infty} s^{m} f_{s}(s) d s / C\right)^{a}} f_{s}(s) d s\right]\right\} d r_{0},
\end{array}
$$

where $f_{r_{0}}\left(r_{0}\right)$ is the PDF of initial strength $r_{0}, n$ represents the load application times, and $f_{s}(s)$ is load PDF. $C$ and $a$ are material parameters that describe the strength degradation process and can be referred to in [21].

In order to consider the relationship between the dynamic reliability and time, the number of load application $n$ in the time duration $t$ is expressed as

$$
n=w(t) t
$$

where $w(t)$ is the frequency function of load. Then the dynamic reliability with respect to time can be further written as

$$
\begin{array}{r}
R_{1}(t)=\int_{0}^{\infty} k\left[1-\int_{0}^{r_{0}} f_{r_{0}}\left(r_{0}\right) d r_{0}\right]^{k-1} f_{r_{0}}\left(r_{0}\right) \\
\cdot\left\{\prod_{i=1}^{w(t) t}\left[\int_{0}^{r_{0}\left(1-i \int_{0}^{\infty} s^{m} f_{s}(s) d s / C\right)^{a}} f_{s}(s) d s\right]\right\} d r_{0} .
\end{array}
$$

The PDF of the series system lifetime can be expressed as follows:

$$
\begin{gathered}
f_{1}(t)=-\frac{\partial}{\partial t} \int_{0}^{\infty} k\left[1-\int_{0}^{r_{0}} f_{r_{0}}\left(r_{0}\right) d r_{0}\right]^{k-1} f_{r_{0}}\left(r_{0}\right) \\
\cdot\left\{\prod_{i=1}^{w(t) t}\left[\int_{0}^{r_{0}\left(1-i \int_{0}^{\infty} s^{m} f_{s}(s) d s / C\right)^{a}} f_{s}(s) d s\right]\right\} d r_{0} .
\end{gathered}
$$

In addition, when the components are assumed to be mutually independent, according to classic reliability theory, the dynamic reliability of independent series system is given by as follows:

$$
\begin{aligned}
R_{2}(t) & =\left[\int_{0}^{\infty} f_{r_{0}}\left(r_{0}\right)\right. \\
\cdot & \left.\left\{\prod_{i=1}^{w(t) t}\left[\int_{0}^{r_{0}\left(1-i \int_{0}^{\infty} s^{m} f_{s}(s) d s / C\right)^{a}} f_{s_{i}}\left(s_{i}\right) d s_{i}\right]\right\} d r_{0}\right]^{k} .
\end{aligned}
$$

The life PDF of the independent series system can be calculated by

$$
\begin{aligned}
f_{2}(t) & =-k\left[\int_{0}^{\infty} f_{r_{0}}\left(r_{0}\right)\right. \\
& \left.\cdot\left\{\prod_{i=1}^{w(t) t}\left[\int_{0}^{r_{0}\left(1-i \int_{0}^{\infty} s^{m} f_{s}(s) d s / C\right)^{a}} f_{s_{i}}\left(s_{i}\right) d s_{i}\right]\right\} d r_{0}\right]^{k-1} \\
& \times \frac{\partial}{\partial t} \int_{0}^{\infty} f_{r_{0}}\left(r_{0}\right) \\
& \cdot\left\{\prod_{i=1}^{w(t) t}\left[\int_{0}^{r_{0}\left(1-i \int_{0}^{\infty} s^{m} f_{s}(s) d s / C\right)^{a}} f_{s_{i}}\left(s_{i}\right) d s_{i}\right]\right\} d r_{0} .
\end{aligned}
$$

Provided that the distributions of residual strength at each load application are adopted in the reliability calculation and SDPD is neglected, (12) can be rewritten as

$$
R_{3}(t)=\left[\prod_{i=1}^{w(t) t} \int_{0}^{\infty} f_{s_{i}}\left(s_{i}\right) \int_{s_{i}}^{\infty} f_{r_{i}}\left(r_{i}\right) d r_{i} d s_{i}\right]^{k},
$$

where $f_{r_{i}}\left(r_{i}\right)$ is the PDF of residual strength at the $i$ th load application. Correspondingly, the life PDF of the series system can be calculated by

$$
\begin{aligned}
& f_{3}(t)=-k\left[\prod_{i=1}^{w(t) t} \int_{0}^{\infty} f_{s_{i}}\left(s_{i}\right)\right. \\
& \left.\cdot \int_{s_{i}}^{\infty} f_{r_{i}}\left(r_{i}\right) d r_{i} d s_{i}\right]^{k-1} \frac{\partial}{\partial t} \prod_{i=1}^{w(t) t} \int_{0}^{\infty} f_{s_{i}}\left(s_{i}\right) \int_{s_{i}}^{\infty} f_{r_{i}}\left(r_{i}\right) d r_{i} d s_{i} .
\end{aligned}
$$

3.2. Residual Lifetime Distribution Models of Mechanical Systems. Provided that a mechanical system still operates normally at the moment $t$, the survival operational time from $t$ is defined as its residual lifetime denoted by $T$. When the randomness of load and material parameters is taken 
into account, it is necessary to obtain the distribution of $T$. The residual lifetime distribution prediction is of great importance to the fault diagnosis, preventive maintenance, and system health management of mechanical products. The PDF of the residual lifetime is essentially the function of the PDF of the whole lifetime of mechanical systems. Thus, the problems of SDPD and failure dependence of components in establishing analytical models for whole lifetime distribution of mechanical systems also bring large difficulties in modeling PDF of the residual lifetime. In this section, the PDFs of residual lifetime of mechanical systems are derived based on the models provided in Section 3.1, which can take the stochastic load process, SDPD, and failure dependence of components into consideration.

According to the conditional probability formula, the PDF of $T$ can be expressed by

$$
f_{T}(T)=\frac{\partial}{\partial T} \frac{\int_{t}^{t+T} f(t) d t}{1-\int_{0}^{t} f(t) d t}
$$

where $f(t)$ is the PDF of the system. Therefore, according to (12), the residual lifetime PDF of the dependent series system can be expressed as follows:

$$
f_{7}(T)=\frac{\partial}{\partial T} \frac{-\int_{t}^{t+T}(\partial / \partial t) \int_{0}^{\infty} k\left[1-\int_{0}^{r_{0}} f_{r_{0}}\left(r_{0}\right) d r_{0}\right]^{k-1} f_{r_{0}}\left(r_{0}\right)\left\{\prod_{i=1}^{w(t) t}\left[\int_{0}^{r_{0}\{1-\Phi(i)\}^{a}} f_{s_{i}}\left(s_{i}\right) d s_{i}\right]\right\} d r_{0} d t}{1+\int_{0}^{t}(\partial / \partial t) \int_{0}^{\infty} k\left[1-\int_{0}^{r_{0}} f_{r_{0}}\left(r_{0}\right) d r_{0}\right]^{k-1} f_{r_{0}}\left(r_{0}\right)\left\{\prod_{i=1}^{w(t) t}\left[\int_{0}^{r_{0}\{1-\Phi(i)\}^{a}} f_{s_{i}}\left(s_{i}\right) d s_{i}\right]\right\} d r_{0} d t} .
$$

From (14), the residual lifetime PDF of the independent series system considering SDPD can be given by

$$
f_{8}(T)=\frac{\partial}{\partial T} \frac{\left\{-\int_{t}^{t+T} k\left[\int_{0}^{\infty} f_{r_{0}}\left(r_{0}\right)\left\{\prod_{i=1}^{w(t) t}\left[\int_{0}^{r_{0}\left(1-i \int_{0}^{\infty} s^{m} f_{s}(s) d s / C\right)^{a}} f_{s_{i}}\left(s_{i}\right) d s_{i}\right]\right\} d r_{0}\right]^{k-1} \times(\partial / \partial t) \int_{0}^{\infty} f_{r_{0}}\left(r_{0}\right)\left\{\prod_{i=1}^{w(t) t}\left[\int_{0}^{r_{0}\left(1-i \int_{0}^{\infty} s^{m} f_{s}(s) d s / C\right)^{a}} f_{s_{i}}\left(s_{i}\right) d s_{i}\right]\right\} d r_{0} d t\right\}}{\left\{1+\int_{0}^{t} k\left[\int_{0}^{\infty} f_{r_{0}}\left(r_{0}\right)\left\{\prod_{i=1}^{w(t) t}\left[\int_{0}^{r_{0}\left(1-i \int_{0}^{\infty} s^{m} f_{s}(s) d s / C\right)^{a}} f_{s_{i}}\left(s_{i}\right) d s_{i}\right]\right\} d r_{0}\right]^{k-1} \times(\partial / \partial t) \int_{0}^{\infty} f_{r_{0}}\left(r_{0}\right)\left\{\prod_{i=1}^{w(t) t}\left[\int_{0}^{r_{0}\left(1-i \int_{0}^{\infty} s^{m} f_{s}(s) d s / C\right)^{a}} f_{s_{i}}\left(s_{i}\right) d s_{i}\right]\right\} d r_{0} d t\right\}} .
$$

The residual lifetime PDF of the independent series system without SDPD taken into account can be written from (16) as follows:

$$
f_{9}(T)=\frac{\partial}{\partial T} \frac{-\int_{t}^{t+T} k\left[\prod_{i=1}^{w(t) t} \int_{0}^{\infty} f_{s_{i}}\left(s_{i}\right) \int_{s_{i}}^{\infty} f_{r_{i}}\left(r_{i}\right) d r_{i} d s_{i}\right]^{k-1}(\partial / \partial t) \prod_{i=1}^{w(t) t} \int_{0}^{\infty} f_{s_{i}}\left(s_{i}\right) \int_{s_{i}}^{\infty} f_{r_{i}}\left(r_{i}\right) d r_{i} d s_{i} d t}{1+\int_{0}^{t} k\left[\prod_{i=1}^{w(t) t} \int_{0}^{\infty} f_{s_{i}}\left(s_{i}\right) \int_{s_{i}}^{\infty} f_{r_{i}}\left(r_{i}\right) d r_{i} d s_{i}\right]^{k-1}(\partial / \partial t) \prod_{i=1}^{w(t) t} \int_{0}^{\infty} f_{s_{i}}\left(s_{i}\right) \int_{s_{i}}^{\infty} f_{r_{i}}\left(r_{i}\right) d r_{i} d s_{i} d t} .
$$

\section{Numerical Examples}

In this section, numerical examples for series mechanical systems are provided to analyze the following problems:

(1) Validation of the proposed models for system lifetime distribution estimation via Monte Carlo simulation (MCS).

(2) The influences of failure dependence of components, SDPD, and initial strength dispersion on whole lifetime distribution of mechanical systems.

(3) The effects of failure dependence of components, SDPD, and initial strength dispersion on residual lifetime distribution of mechanical systems.

4.1. Monte Carlo Simulation Validation. Owing to the operational mechanism of mechanical products, mechanical components in mechanical systems are always logically connected in series configuration. The practical examples of series mechanical systems and parallel systems can be referred to in [21]. In this section, for illustrative convenience, it is assumed that the components are identical in the series system shown in Figure 12. The statistical parameters of load and material parameters in [21] are adopted for the components in the two systems, which are listed in Table 1. To validate the lifetime distribution models, MCSs are performed with the flow chart shown in Figure 7. In the MCS, the deterministic residual strength under deterministic load is calculated according to the strength degradation rule in physical experiment with respect to component cumulative damage $D(n)$ as follows [21]:

$$
r(n)=r[1-D(n)]^{a}
$$




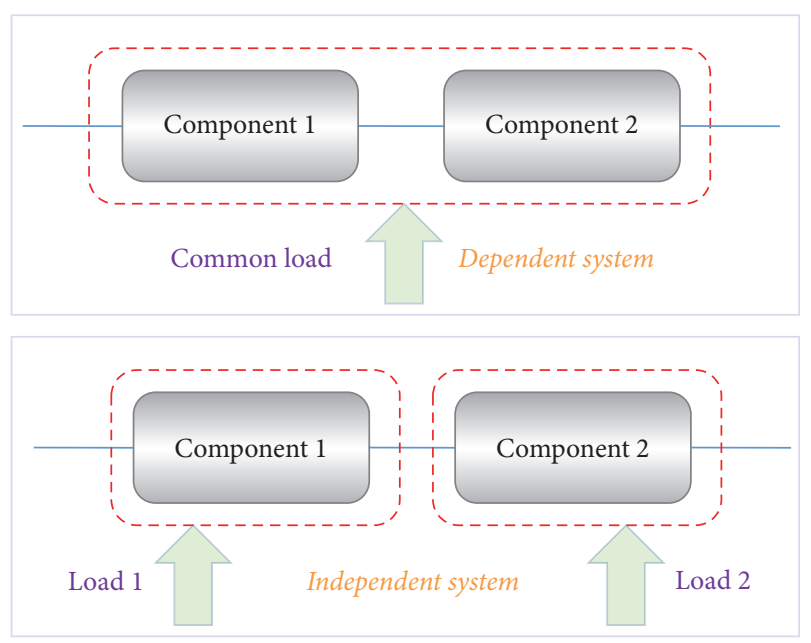

FIGURE 12: Series mechanical systems.

TABLE 1: Stress parameters and material parameters of explosive bolts.

\begin{tabular}{lccccccc}
\hline $\begin{array}{l}\mu\left(r_{0}\right) \\
{[\mathrm{MPa}]}\end{array}$ & $\begin{array}{c}\sigma\left(r_{0}\right) \\
{[\mathrm{MPa}]}\end{array}$ & $\begin{array}{c}\mu(s) \\
{[\mathrm{MPa}]}\end{array}$ & $\begin{array}{c}\sigma(s) \\
{[\mathrm{MPa}]}\end{array}$ & $m$ & $a$ & $\begin{array}{c}w(t) \\
{\left[\mathrm{h}^{-1}\right]}\end{array}$ & $\begin{array}{c}\mathrm{C} \\
{\left[\mathrm{MPa}^{2}\right]}\end{array}$ \\
\hline 400 & 30 & 300 & 20 & 2 & 1 & 0.5 & $10^{8}$ \\
\hline
\end{tabular}

Hence, the MCS, independent of any analytical model, is essentially identical with physical experiment. The results from the Monte Carlo simulation and the system lifetime PDF calculated by using the proposed models are shown in Figure 13.

From Figure 13, it can be seen that the lifetime PDF obtained from the proposed model shows good agreement with the lifetime PDF from MCS. The model developed in this paper provides a theory basis for analytical method to quantitative lifetime distribution assessment. In addition, unlike the normal distribution curve, short system lifetime accounts for a certain proportion of the lifetime distribution, which is analogous to the situation in the infant mortality period of the bathtub curve.

In addition, in order to illustrate the error in lifetime distribution estimation by using conventional RCF method in the case of nonergodic and nonstationary stress process, the time-dependent mean value of the stress process is assumed to obey the following form:

$$
\mu_{s}(t)=300(1+0.0005 t) .
$$

Then, the comparison between the lifetime distribution of the dependent system by means of the proposed method and the lifetime distribution based on conventional RCF method with the PPDF of stress obtained via the statistics of the MCSs in this section is shown in Figure 14.

From Figure 14, it can be seen that large calculation error in system lifetime distribution estimation could be caused due to the usage of conventional RCF method when the stress is nonergodic or nonstationary. The incorrect usage of the conventional RCF method could result in the underestimation of the system lifetime distribution in the

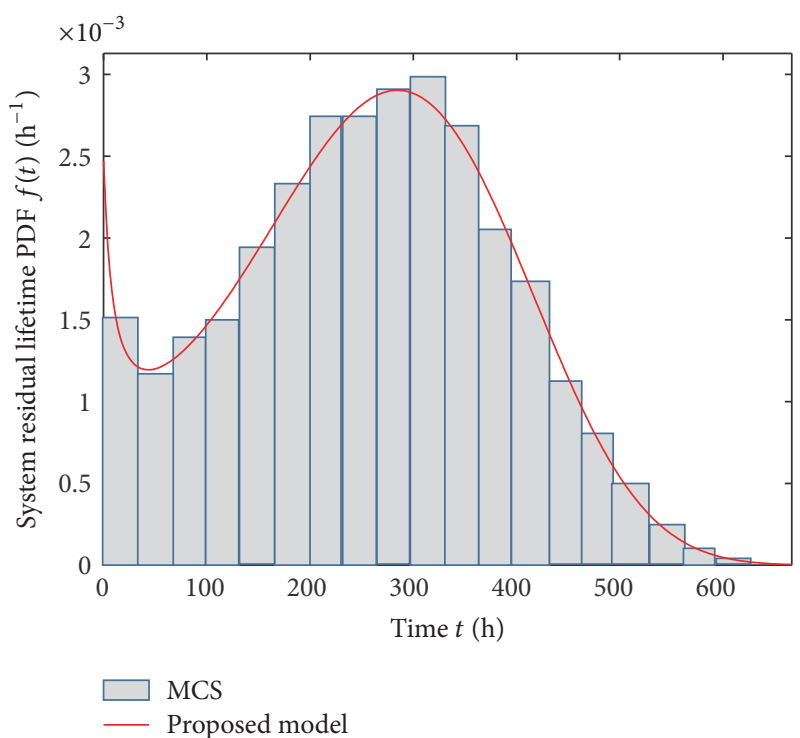

FIGURE 13: Lifetime PDF from MCS and lifetime PDF from proposed models.

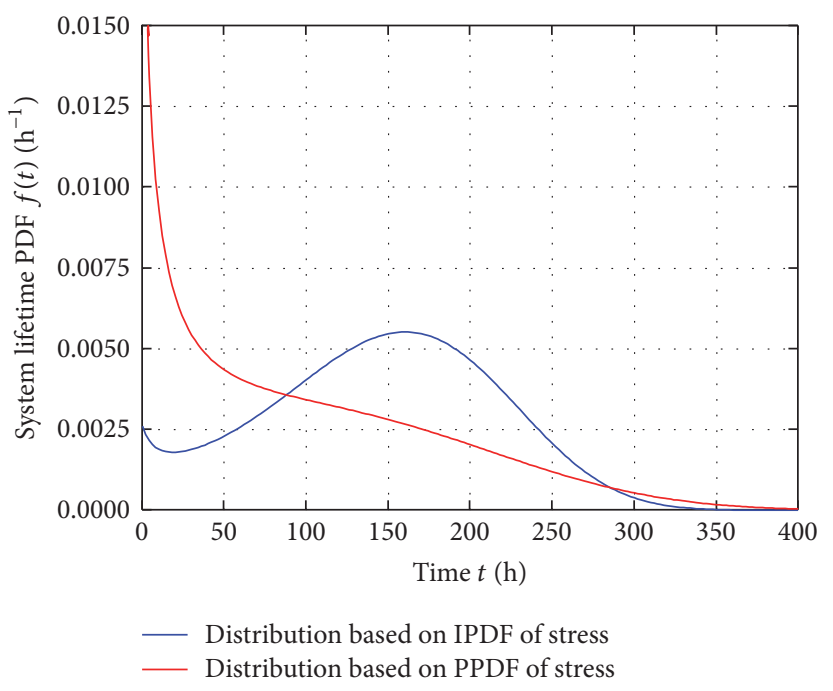

Figure 14: System lifetime PDF based on different PDF of stress.

long lifespan regions. Furthermore, the increase in the mean value of the stress substantially reduces the system lifetime.

4.2. The Effects of SDPD and Failure Dependence of Components on System Whole Lifetime Distribution. For the series system in Section 4.1, when the components are assumed to be mutually independent, the system lifetime PDF is shown in Figure 15. Besides, provided that the strength distribution at each load application is used for lifetime distribution assessment without SDPD taken into account, the system lifetime PDF is also shown in Figure 15. In addition, when the series system is composed of four components, the system lifetime PDFs are shown in Figure 16. Moreover, in the cases of different initial strength dispersion, the lifetime PDFs 


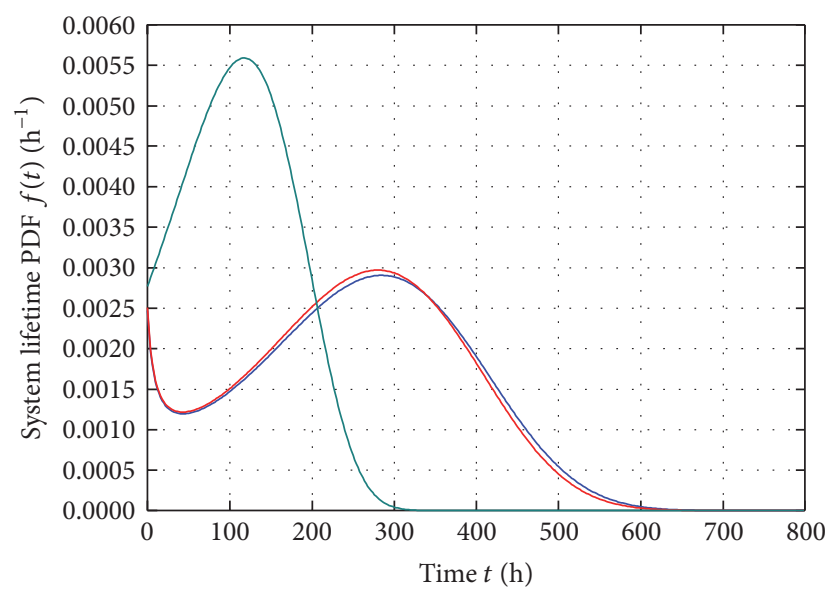

- Dependent system considering SDPD

— Independent system considering SDPD

_ Independent system without considering SDPD

Figure 15: System lifetime PDF with two components.

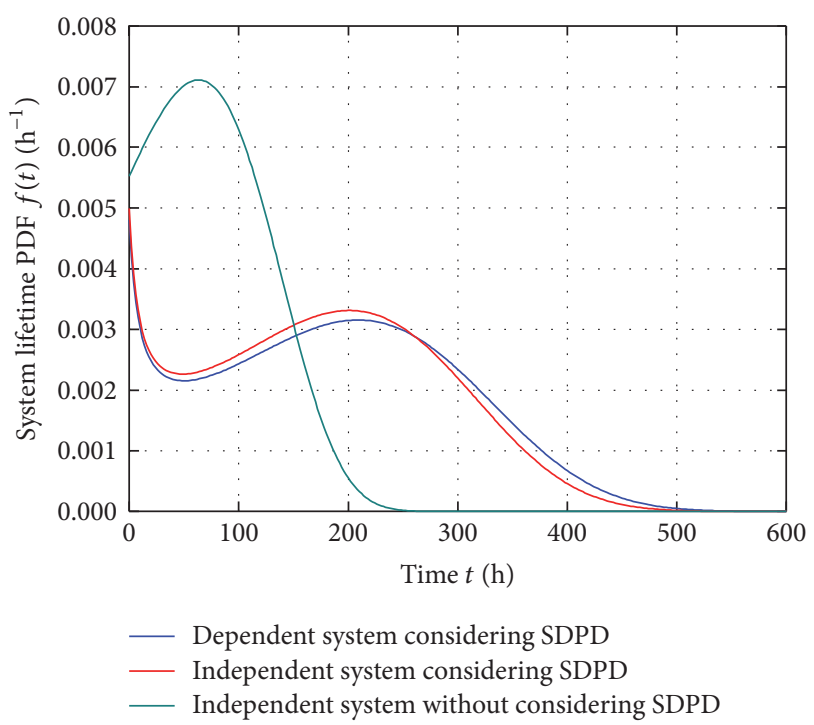

FIGURE 16: System lifetime PDF with four components.

of the dependent system considering SDPD are shown in Figure 17.

From Figures 15-17, it can be seen that the lifetime of dependent series systems is distributed in the interval of longer lifetime compared with the lifetime of independent systems. The proposed system lifetime distribution models provide the analytical methods for system lifetime distribution assessment considering the failure dependence of components in an explicit form. The conventional system lifetime distribution models, in which components are assumed to be mutually independent, could lead to error in system lifetime distribution evaluation. Moreover, most mechanical systems are in the series configuration, such as the drive systems and the mechanisms for motion transmission. The series

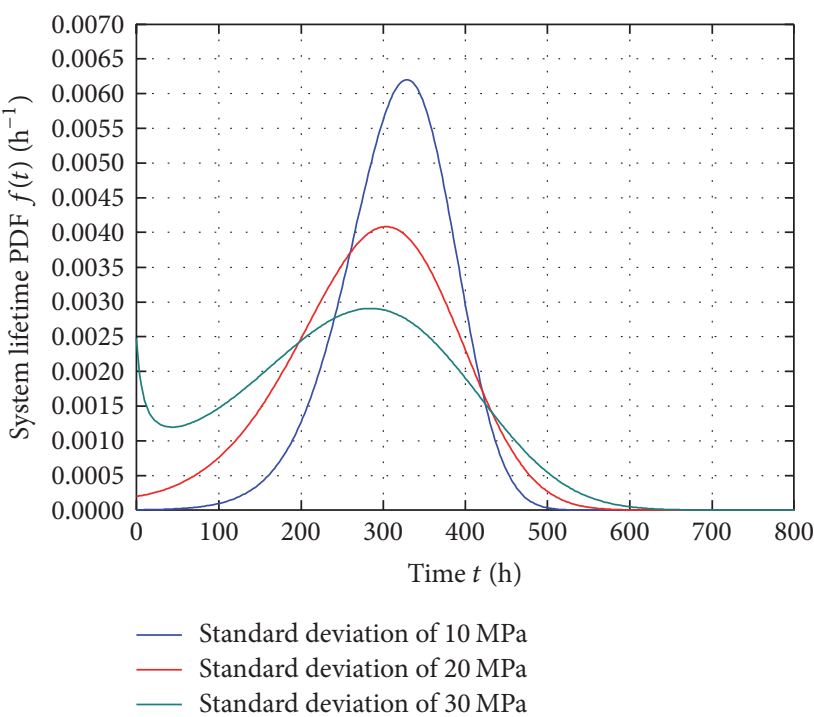

FIGURE 17: System lifetime in cases of different strength dispersion.

systems might be composed of a large amount of components. From Figures 15 and 17, it can be learnt that the increase in the number of components in the series systems results in the enhancement of the effects of failure dependence on system lifetime distribution. Thus, more attention should be paid to system lifetime distribution in the case where series systems comprise a larger amount of components. Furthermore, SDPD has much more significant influences on system lifetime distribution than failure dependence of components. The mean value and dispersion of system lifetime could be underestimated due to neglecting the phenomenon of SDPD. In addition, the dispersion of initial strength also has great impacts on system lifetime distribution. In general, the decrease in the dispersion of initial strength makes the system lifetime distribution more concentrated and causes the increase in the mean value of system lifetime.

4.3. The Impacts of SDPD and Failure Dependence of Components on System Residual Lifetime Distribution. For the dependent series system in Section 4.1, the system residual lifetime PDFs from different time instant $t$ are shown in Figure 18. Besides, in the cases of different initial strength dispersion, the residual lifetime PDFs of the dependent system from $t=100$ are shown in Figure 19. In addition, the residual lifetime PDFs of independent system from $t=100$ considering SDPD and that without SDPD taken into account are shown in Figure 20.

It is difficult to quantitatively assess system residual lifetime distribution via an explicit mathematical expression with the failure dependence of components, SDPD, and the statistical characteristics of both load process and material parameters into consideration. To address these difficulties, analytical models for system residual lifetime distribution are proposed in this paper. From Figures 18-20, it can be learnt that the failure dependence, SDPD, and statistical characteristics of material parameters have considerable impacts 


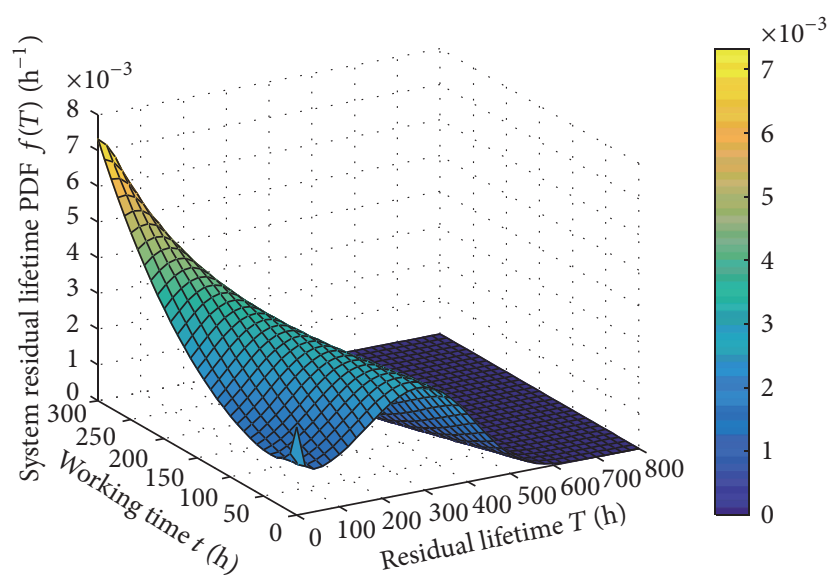

FIGURE 18: System residual lifetime PDF at different moment.

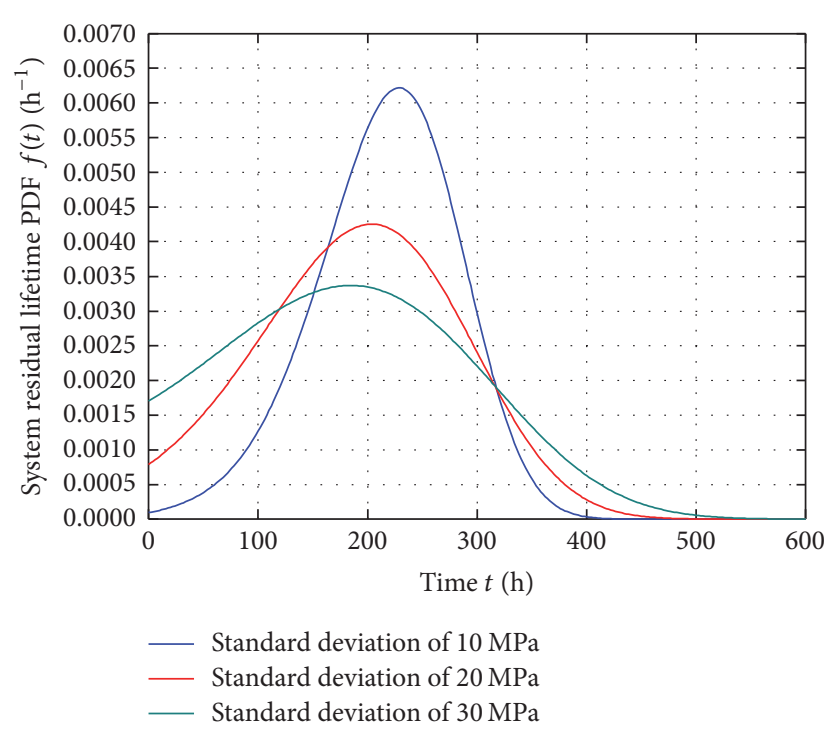

FIGURE 19: System residual lifetime PDF in cases of different strength dispersion.

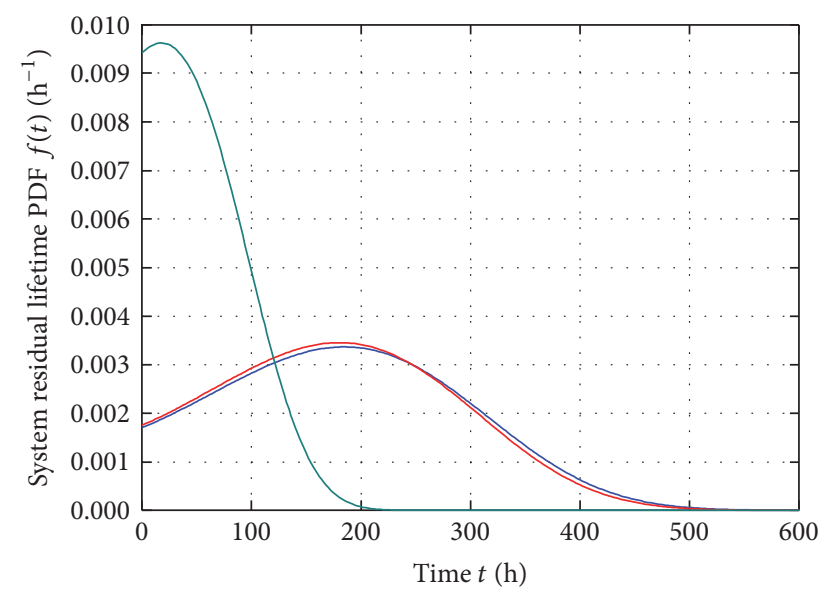

— Dependent system considering SDPD

— Independent system considering SDPD

_ Independent system without considering SDPD

Figure 20: System residual lifetime PDF. on system residual lifetime distribution. The shape of the residual lifetime PDF varies with the increase in the working time. Generally, the peak value of the residual lifetime PDF increases and moves towards the left direction with the increase in the working time. After working for a certain period of time, the local extremum of residual lifetime PDF in the interval of short residual lifetime disappears. Besides, compared with the failure dependence of components, SDPD have more significant influences on residual lifetime distribution. The system lifetime could be underestimated without SDPD taken into consideration. In addition, the increase in the initial strength dispersion results in larger dispersion and smaller mean value of system residual lifetime.

\section{Conclusions}

For components in the series mechanical systems, the lifetime distribution is determined by both the load process and strength degradation process. When the load process is not ergodic or stationary, conventional RCF method should be used with caution, which could lead to error in lifetime distribution estimation of components and systems. Moreover, SDPD results from the dynamic interaction between the load process and strength degradation process. It could cause large error in lifetime distribution assessment of components and systems to neglect the phenomenon of SDPD. In addition, SDPD and failure dependence of components jointly bring considerable difficulties in prediction of system lifetime distributions and system residual lifetime distributions.

To deal with these problems, reliability-based analytic models for estimation of whole lifetime distribution and residual lifetime distribution of series mechanical systems under random load are developed in this paper, which take the time-dependent statistical parameters of load process and strength degradation process as the input of the models and consider the dynamic interaction between load process and strength degradation process. Moreover, SDPD and failure dependence of components are taken into account in the proposed models in an explicit mathematical expression. The results show that SDPD has significant influences on system lifetime distribution. The mean value and dispersion of system lifetime could be underestimated due to neglecting the phenomenon of SDPD. Furthermore, increase in the number of components in the series systems results in the enhancement of the effects of failure dependence on system lifetime distribution. Compared with the failure dependence of components, SDPD have more significant influences on system lifetime distribution. Besides, decrease in the dispersion of initial strength makes the system lifetime distribution more concentrated and causes the increase in the mean value of system lifetime. In addition, the shape of the residual lifetime PDF varies with the increase in the working time.

\section{Conflicts of Interest}

The authors declare that there are no conflicts of interest regarding the publication of this article. 


\section{Authors' Contributions}

All authors have read and approved this version of the article, and due care has been taken to ensure the integrity of the work.

\section{Acknowledgments}

This work is supported by National Natural Science Foundation of China (Grant no. 51505207), Scientific Research Fund of Liaoning Provincial Education Department of China (Grant no. L2015298), Liaoning Provincial Natural Science Foundation of China (Grant no. 2015020152), and Open Foundation of Zhejiang Provincial Top Key Academic Discipline of Mechanical Engineering (Grant no. ZSTUME02A01).

\section{References}

[1] G. Hao and H. Li, "Conceptual designs of multi-degree of freedom compliant parallel manipulators composed of wire-beam based compliant mechanisms," Proceedings of the Institution of Mechanical Engineers, Part C: Journal of Mechanical Engineering Science, vol. 229, no. 3, pp. 538-555, 2015.

[2] J. Wu and S. Yan, "An approach to system reliability prediction for mechanical equipment using fuzzy reasoning Petri net," Proceedings of the Institution of Mechanical Engineers, Part O: Journal of Risk and Reliability, vol. 228, no. 1, pp. 39-51, 2014.

[3] Y. Zhao, "A fatigue reliability analysis method including super long life regime," International Journal of Fatigue, vol. 35, no. 1, pp. 79-90, 2012.

[4] S. Suresh, Fatigue of Materials, Cambridge University Press, 1998.

[5] T. I. Elperin and I. B. Gertsbakh, "Estimation in a random censoring model with incomplete information: exponential lifetime distribution," IEEE Transactions on Reliability, vol. 37, no. 2, pp. 223-229, 1988.

[6] Z. Pan, J. Feng, and Q. Sun, "Lifetime distribution and associated inference of systems with multiple degradation measurements based on gamma processes," Eksploatacja i Niezawodnosc - Maintenance and Reliability, vol. 18, no. 2, pp. 307-313, 2016.

[7] A. Asgharzadeh, M. Abdi, and S.-J. Wu, "Interval estimation for the two-parameter bathtub-shaped lifetime distribution based on records," Hacettepe Journal of Mathematics and Statistics, vol. 44, no. 2, pp. 399-416, 2015.

[8] M. Kayid and S. Izadkhah, "Characterizations of the exponential distribution by the concept of residual life at random time," Statistics \& Probability Letters, vol. 107, pp. 164-169, 2015.

[9] J. Gong, Y.-F. Fu, W. Xia, J.-H. Li, and F. Zhan, "Fatigue life prediction of screw blade in screw sand washing machine under random load with gauss distribution," American Journal of Engineering and Applied Sciences, vol. 9, no. 4, pp. 1198-1212, 2016.

[10] Y. Mori and B. R. Ellingwood, “Time-dependent system reliability analysis by adaptive importance sampling," Structural Safety, vol. 12, no. 1, pp. 59-73, 1993.

[11] M. G. Stewart and D. V. Rosowsky, "Time-dependent reliability of deteriorating reinforced concrete bridge decks," Structural Safety, vol. 20, no. 1, pp. 91-109, 1998.
[12] R. E. Glaser, "Bathtub and related failure rate characterizations," Journal of the American Statistical Association, vol. 75, no. 371, pp. 667-672, 1980.

[13] G. Stefanou and M. Papadrakakis, "Stochastic finite element analysis of shells with combined random material and geometric properties," Computer Methods Applied Mechanics and Engineering, vol. 193, no. 1-2, pp. 139-160, 2004.

[14] M. Ostoja-Starzewski and X. Wang, "Stochastic finite elements as a bridge between random material microstructure and global response," Computer Methods Applied Mechanics and Engineering, vol. 168, no. 1-4, pp. 35-49, 1999.

[15] B. N. Singh, D. Yadav, and N. G. R. Iyengar, "Natural frequencies of composite plates with random material properties using higher-order shear deformation theory," International Journal of Mechanical Sciences, vol. 43, no. 10, pp. 2193-2214, 2001.

[16] T.-H. Lee and K. M. Mosalam, "Seismic demand sensitivity of reinforced concrete shear-wall building using FOSM method," Earthquake Engineering \& Structural Dynamics, vol. 34, no. 14, pp. 1719-1736, 2005.

[17] J. W. Baker and C. A. Cornell, Uncertainty Specification And Propagation for Loss Estimation Using FOSM Method, Pacific Earthquake Engineering Research Center, College of Engineering, University of California, 2003.

[18] C. Guohua and D. Shuho, "Study on the reliability assessment methodology for pressure vessels containing defects," International Journal of Pressure Vessels and Piping, vol. 69, no. 3, pp. 273-277, 1996.

[19] Y. G. Zhao, T. Ono, and M. Kato, "Second-order third-moment reliability method," Journal of Structural Engineering, vol. 128, no. 8, pp. 1087-1090, 2002.

[20] Z. P. Qiu and J. Wang, "The interval estimation of reliability for probabilistic and non-probabilistic hybrid structural system," Engineering Failure Analysis, vol. 17, no. 5, pp. 1142-1154, 2010.

[21] P. Gao, L. Xie, and F. Liu, "Dynamic reliability analysis of series mechanical systems considering strength degradation path dependence of components," Journal of Advanced Mechanical Design, Systems, and Manufacturing, vol. 9, no. 5, pp. 1-13, 2015. 


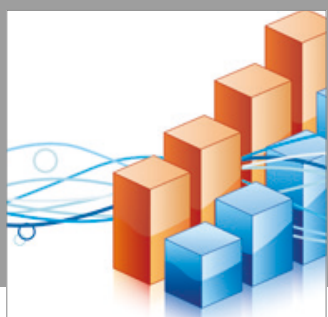

Advances in

Operations Research

vatersals

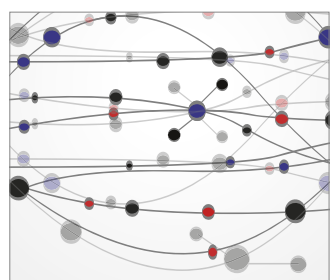

\section{The Scientific} World Journal
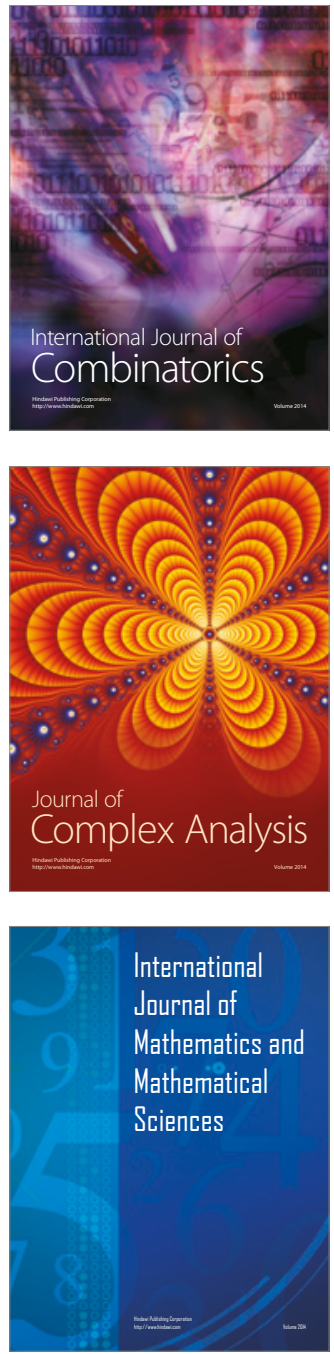
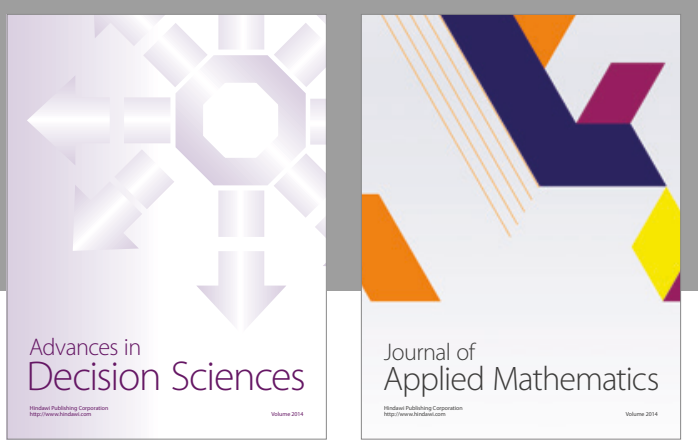

Algebra

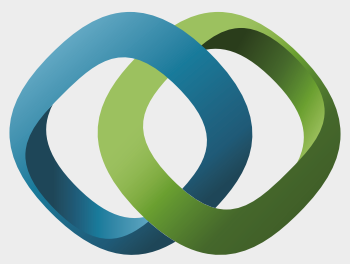

\section{Hindawi}

Submit your manuscripts at

https://www.hindawi.com
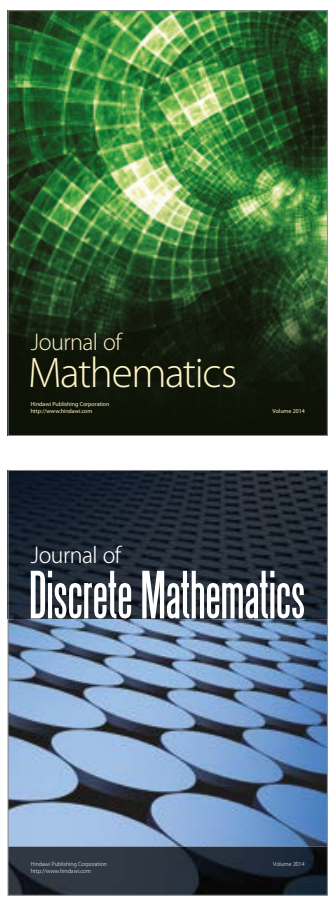

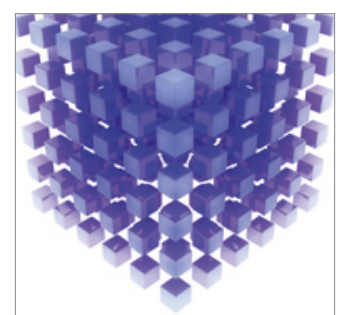

Mathematical Problems in Engineering
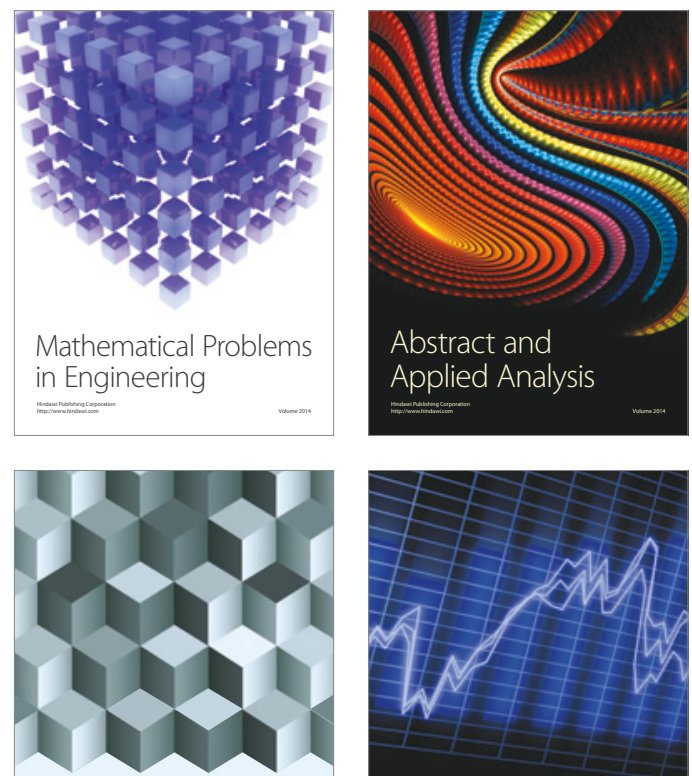

Journal of

Function Spaces

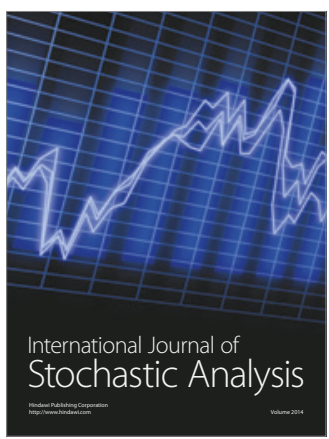

Probability and Statistics
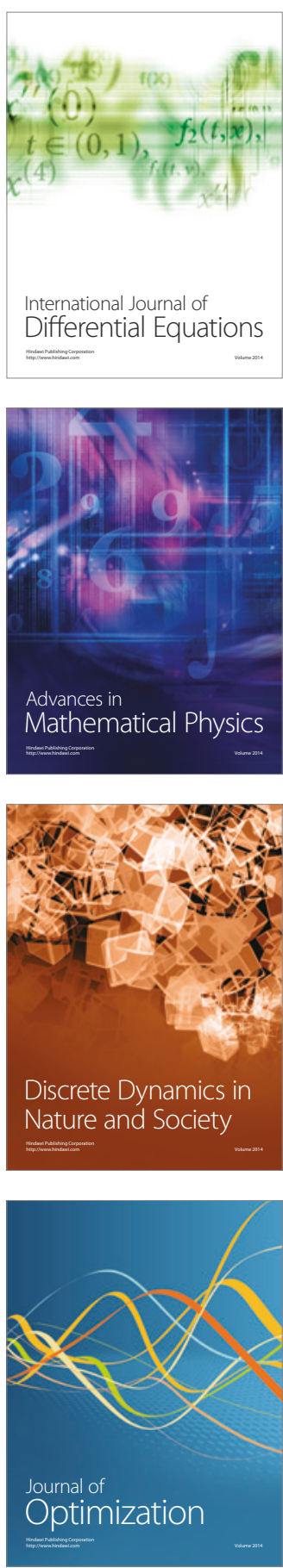